\title{
Hemorrhagic Stroke in a Dog with Anaplastic Carcinoma of the Mammary Gland
}

\author{
Bianca Santana de Cecco', Marina Paula Lorenzett', Thainã Piccolo Vargas' ${ }^{1}$, Júlia Gabriela Wronski', \\ Marcia Düster², Franciele Maboni Siqueira ${ }^{3}$, Saulo Petinatti Pavarini' \& David Driemeier'
}

\begin{abstract}
Background: Anaplastic carcinoma of the mammary gland is considered a highly invasive and malignant neoplasia, which usually leads to neoplastic thrombosis and epidermal ulceration due to invasiveness of lymphatic vessels by tumor emboli. Loss of epidermal barrier predisposes to secondary bacterial infection, mainly by commensal bacteria of the skin, and septicemia can be observed in cases of systemic dissemination. Neoplastic emboli and septic thrombi can lead to vascular obstruction, which can predispose to infarction in multiple organs. The aim of this case report is to describe a case of hemorrhagic stroke in a dog with anaplastic carcinoma of the mammary gland.

Case: A 12-year-old, mixed breed, female spayed canine presented with mammary tumor with plaque formation, associated with skin ulceration which extended to the medial region of pelvic limbs. The animal also displayed neurologic clinical signs characterized by stupor, opisthotonos and apathy. Due to poor prognosis, the canine was euthanized and submitted to necropsy. During external examination, the animal was in a good body condition, had moderately pale mucosae, and it was noted a nodular plaque in the abdominal ventral area extending from thoracic to inguinal mammary gland. The mass was firm and white, extensively ulcerated, and extending from the thoracic to inguinal mammary gland. There was a well circumscribed area of infarction in the spleen and kidney. In the cerebral cortex it was observed an extensive area of hemorrhage with adhered blood clot from frontal to temporal lobe. There were multiple well circumscribed, firm, and white areas in the myocardium. Histologically, the neoplastic proliferation was composed by epithelial cells with high pleomorphism, features of malignancy, and no delimitations, leading to the diagnosis of anaplastic carcinoma of the mammary gland. Admixed with tumor cells there were multiple areas of hemorrhage, necrosis and thrombosis. In the cerebral cortex there was a focally extensive area of hemorrhage, multifocal thrombosis, abundant amount of gitter cells, and moderate perivascular inflammatory infiltrate of lymphocytes. There was neoplastic infiltrate in the bladder, adrenal glands, and lungs. There were multiple areas of necrosis and thrombosis in spleen and heart. Bacterial examination was performed in fragments of cerebral cortex and kidney, and Staphylococcus pseudintermedius was isolated.

Discussion: Anaplastic carcinoma of the mammary gland has an epithelial origin and is considered the most malignant neoplasm with worst prognosis due to its characteristic of high cellular pleomorphism and the ability of invasiveness of vessels and adjacent tissues. Therapeutic options are scarce and palliative, and surgical removal is not indicated. Dermal ulceration usually is infected by opportunistic bacteria that can lead to inflammation and pain, and treatment in these cases is focused on pain management with analgesics. The bacterial infection can evolve to septicemia with decrease of endogenous anticoagulants and consequent blood hypercoagulability. Moreover, studies demonstrate the higher risk of clotting abnormalities when associated to progression of mammary carcinomas, which includes anaplastic carcinoma of the mammary gland. In this study, two causes which may have led to thromboembolism and cerebral hemorrhage are discussed: septicemia after invasion of lymphatic vessels by the anaplastic carcinoma or hypercoagulability caused by the systemic neoplasia, which could have reduce the blood flow to vessels of the cerebral cortex.
\end{abstract}

Keywords: canine, thrombosis, neoplasm, cerebral infarction. 


\section{INTRODUCTION}

Anaplastic carcinoma is the most malignant mammary carcinoma of dogs and is characterized by marked invasion of tissues and lymphatic vessels, associated to high metastasis index [6]. This tumor may present with paraneoplastic syndrome such as hypercoagulability and secondary thromboembolism [4]. Furthermore, because of its characteristics of lymphatic vessels invasiveness, this type of carcinoma can cause epidermal ulceration and predispose to secondary bacterial infection by commensal bacteria of the skin [6] Sepsis can be developed after bacterial infection, with reduction of endogenous anticoagulants responsible for prevention of thrombosis, which also leads to higher risk of thromboembolism [5]. The aim of this case report is to describe a case of anaplastic carcinoma of the mammary gland which caused hemorrhagic stroke in the cerebral cortex of a canine.

\section{CASE}

A 12-year-old, mixed breed, female spayed canine was referred to the small animal clinical service with history of mammary tumor with evolution of months, and ulcerated wounds for 30 days. The tutor was treating the canine at home with healing ointments and homemade herbal formulations. The animal did not improve clinically, subsequently developing vomit with blood and neurological clinical signs characterized by opisthotonos, stupor and frequent vocalization.

Physical examination revealed pale ocular and oral mucosa, prolonged capillary perfusion time, hypothermia, weak pulse, and moderate dehydration. The animal arrived needing emergency care, and was submitted to treatment aiming stabilization of vital signs, analgesia and intense antibiotic therapy. However, the dog continued vomiting blood and died. Necropsy examination was performed with permission of the owner.

At necropsy, the animal had a large tumor extending from thoracic to inguinal mammary glands, presented as plaque formation with elevated surface, firm at palpation and with multiple nodules, which were white with red at cut surface. Associated to this area and continuing to the medial surface of pelvic limbs, there was multiple skin ulceration correlated to abundant fibrin deposition (Figure 1). In pelvic limbs, there was also moderate and diffuse swelling.
At internal examination, inguinal lymph nodes were enlarged, firm and white. Multiple well-circumscribed pale areas were observed in the myocardium. Spleen and kidney had multiple red and well-defined areas characteristics of infarction. In the cerebral cortex there was a focally extensive area with blood clot formation which extended from frontal to temporal region, associated to softness of the parenchyma (Figure 2).

Histological examination showed a mammary neoplasia consisting on a proliferation of highly pleomorphic epithelial cells, non-delimited and non-encapsulated, organized as individualized groups, and occasionally forming papillary projections interspersed with marked fibrovascular stroma (Figure 3). These cells presented several malignancy features including marked anisocytosis and anisokaryosis, karyomegaly and coarse chromatin. Admixed with the tumor there was focally extensive area of ulceration with abundant inflammatory infiltrate composed by neutrophils and macrophages, extensive necrosis, thrombosis, hemorrhage and several basophilic coccoid bacterial aggregates. Similar neoplastic cells were observed in lymph nodes, urinary bladder, adrenal gland and lungs.

Multiple areas of necrosis associated to thrombosis, hemorrhage and a small number of bacteria aggregates were noted in the spleen, liver, kidney and heart. In the stomach there were areas of necrosis admixed with fibrin deposition and inflammatory infiltrate of degenerated neutrophils, besides multifocal thrombosis. In the brain, the hemorrhagic areas were associated with abundant amount of gitter cells, perivascular neutrophils, as well as areas of gliosis and thrombosis (Figure 4). Definitive diagnosis was established as anaplastic carcinoma of the mammary gland (grade III) with lymphatic invasion, metastasis and septicemia.

Refrigerated samples of cerebral cortex and kidney were forwarded to bacteriological analysis, where culture in aerobiosis was performed, as well as biochemical identification and confirmation by MALDI-TOF spectrometry (Microflex LT) ${ }^{1}$, identifying the bacteria Staphylococcus pseudintermedius.

\section{DISCUSSION}

Anaplastic carcinoma of the mammary gland is the most malignant epithelial tumor of dogs and is usually related to invasiveness of lymphatic vessels mainly from the skin [6]. In a study of neoplasms me- 


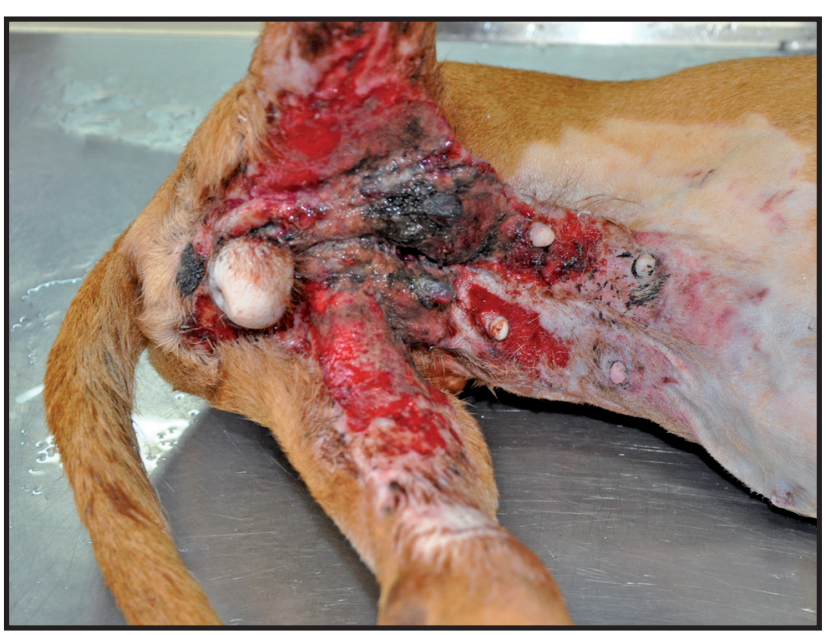

Figure 1. Anaplastic carcinoma of the mammary gland in a canine. Neoplastic plaque formation at inguinal mammary gland, as well as extensive areas of epidermal ulceration.

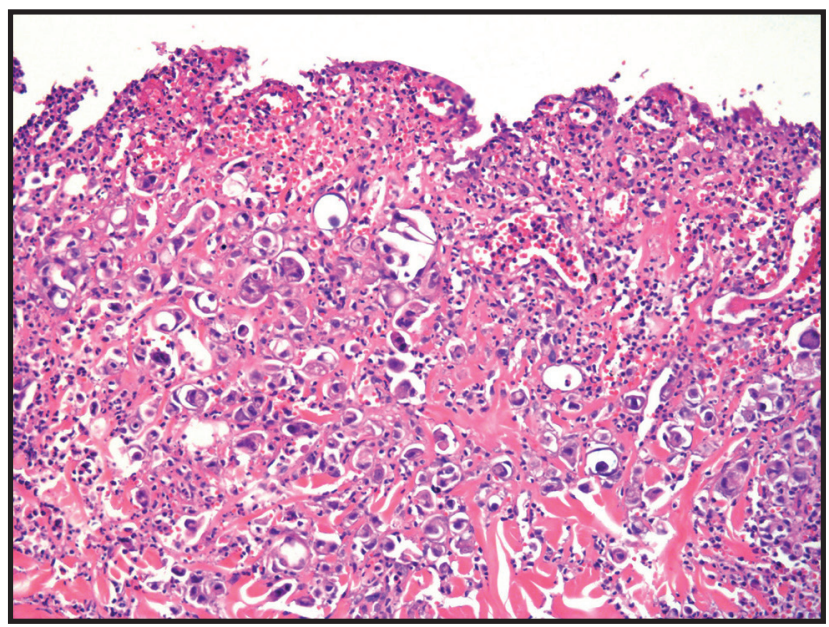

Figure 3. Anaplastic carcinoma of the mammary gland in a canine. Neoplastic proliferation of anaplastic epithelial cells, with arranged in isolated pattern and interspersed by abundant fibrovascular stroma. There is marked cellular atypia demonstrated by marked anisocytosis and karyomegaly. Focally extensive area of ulceration with abundant inflammatory infiltrate composed by neutrophils [HE; Bar= $50 \mu \mathrm{m}]$.

tastasizing to central nervous system, it was demonstrated that anaplastic carcinoma was the main responsible for metastasis among all mammary tumors, and it is directly connected to its behavior of invasiveness and malignancy [7]. Clinically, anaplastic carcinoma is characterized as an inflammatory carcinoma, since the dissemination includes superficial dermis and epidermis, which results in ulceration, suppurative inflammation, and possible bacterial contamination [6]. Tumoral dissemination results in blockage of lymphatic vessels from dermis by neoplastic emboli, leading to infarction due to anoxia and marked edema [6]. In the case here described, there was swelling of limbs associated to epidermal ulceration in the medial aspect of pelvic limbs, working as a portal of entry to bacterial infection.

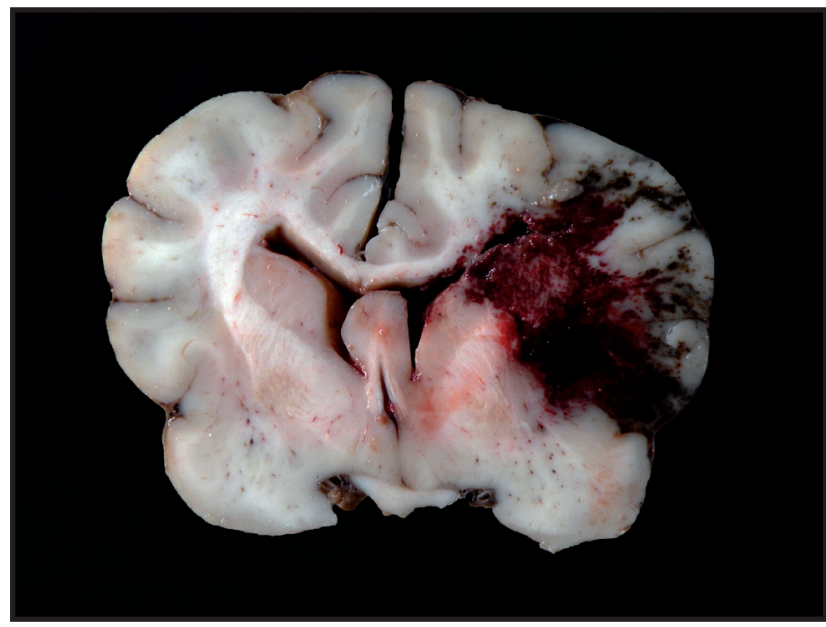

Figure 2. Hemorrhagic stroke. Brain. Extending from parietal to temporal lobe, there is a focally extensive area of hemorrhage associated to blood clot deposition.

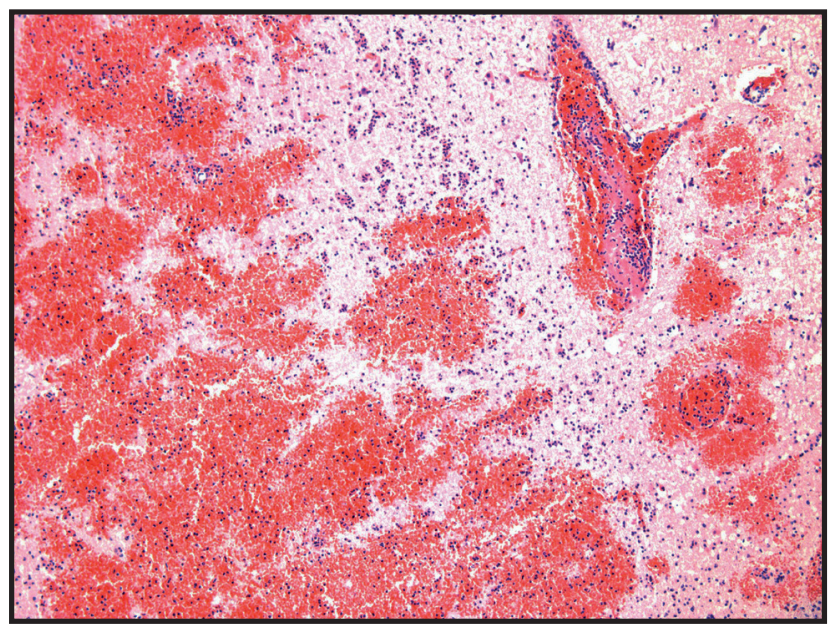

Figure 4. Hemorrhagic stroke. Brain. Focally extensive area of hemorrhage associated to perivascular infiltrate of neutrophils, as well as multifocal areas with gitter cells and macrophages in erytrophagia [HE; Bar $=200 \mu \mathrm{m}$ ].

Bacterial infection of epidermis can be caused by commensal bacteria, such as Staphylococcus pseudintermedius, which was isolated on this case. $S$. pseudintermedius is a commensal bacterium of skin and mucosa of dogs usually classified as an opportunistic agent, however it may cause disease when alterations at epidermal barrier, immunosuppression, and atopic dermatitis occur [1]. S. pseudintermedius secretes toxins that can damage the host cell membrane, such as pore-forming toxins like hemolysins, necrotic and apoptotic toxin of polymorphonuclear and mononuclear cells like Panton-Valentine leucocidin (PVL) toxin [12], therefore, these factors usually causes skin or soft tissue infections and necrotizing infections. Bacterial invasion of tissues and hematogenous dissemination may lead to sepsis, which is considered a systemic response to infection, and it is characterized by mobilization of 
pro-inflammatory cytokines and cells of the immune system, such as neutrophils and macrophages [9].

Release of pro-inflammatory cytokines and expression of tissue factors may stimulate the clotting cascade, regulation of fibrin degradation system and decrease of endogenous anticoagulants, such as antithrombin and C-protein [5]. The role of these endogenous anticoagulants is the thrombus regulation, however in cases of sepsis both of them can be decreased due to excessive consumption, predisposing to thromboembolism $[5,9]$. Not only these cytokines, but also cancer cells can be prothrombotic themselves, inducing the production of thrombin which lead to fibrinogen generation and downregulation of fibrinolysis, that can induce a procoagulant state [10].

Thromboembolism is a vascular obstruction originated from a distant vascular site. One of the main causes of thromboembolism is the hypercoagulability state, which can be a consequence of hemolytic anemia, sepsis, hyperadrenocorticism and systemic neoplasia [2]. The mechanisms to the development of hypercoagulability are multifactorial, and may affect all of Virchow's triad components [10]. In humans, the second leading cause of death with cancer are thrombosis and hypercoagulability; consequently, thrombosis related to neoplasia are extensively studied and protocols with anticoagulation drugs are well established $[3,10]$.

In dogs, studies demonstrate the higher risk of clot abnormalities when associated to progression of mammary carcinomas [8]. Studies in veterinary medicine demonstrates that neoplasia were a common concurrent condition associated to thrombosis, and in dogs with distant metastasis had significantly higher fibrinogen and D-dimer concentrations compared to dogs with local disease [10,11]. Cerebral infarction, also known as stroke, is a common condition that occur secondary to reduction of cerebral blood flow that can be caused by thrombosis and/or hypoperfusion. Although hypercoagulability is described as the cause of strokes, there is little information in veterinary medicine investigating the role of thrombosis in cerebral infarction [10].

Based on clinical history, necropsy findings, histopathology and bacteriology, we diagnosed septicemia due to Staphylococcus pseudintermedius infection developed secondarily to anaplastic carcinoma of the mammary gland resulting on massive brain hemorrhage. This commensal bacterium of the skin resulted on sepsis most likely due to multiple epidermal ulcerations arising from the mammary carcinoma. However, it should be noted that besides bacterial septicemia, the metastatic neoplasia may also result in hypercoagulability state, which can contribute to thrombosis and resultant infarctions followed by hemorrhage.

\section{MANUFACTURER}

${ }^{1}$ Microflex LT. Bruker Industry, Leipzig, Germany.

Declaration of interest. The authors report no conflicts of interest. The authors alone are responsible for the content and writing of paper.

\section{REFERENCES}

1 Bannoehr J. \& Guardabassi L. 2012. Staphylococcus pseudintermedius in the dog: taxonomy, diagnostics, ecology, epidemiology and pathogenicity. Veterinary Dermatology. 23: 253-e52.

2 Boudreau C.E. 2017. An update on cerebrovascular disease in dogs and cats. Veterinary clinics of North America: Small Animal Practice. 48: 45-62.

3 Buller H.R., Van Doormaal F.F., Van Sluis G.L. \& Kamphuisen P.W. 2007. Cancer and thrombosis: from molecular mechanisms to clinical presentation. Journal of thrombosis and haemostasis. 5(1): 246-254.

4 Duda N.C.B., Valle S.F., Matheus J.P., Angeli N.C., Vieira L.C., Oliveira L.O., Sonne L. \& González F.H.D. 2017. Paraneoplastic hematological, biochemical, and hemostatic abnormalities in female dogs with mammary neoplasms. Pesquisa Veterinária Brasileira. 37(5): 479-484.

5 Fourrier F., Chopin C., Goudemand J., Henrycx S., Caron C., Rime A., Marey A. \& Lestavel P. 1992. Septic shock, multiple organ failure, and disseminated intravascular coagulation: Compared patterns of antithrombin III, protein C, and protein S deficiencies. Chest. 101: 816-823.

6 Goldschmidt M.H., Peña L. \& Zappulli V. 2017. Tumors of the mammary gland. In: Tumors in domestic animals. 5th edn. Ames: John Wiley \& Sons, pp.723-765.

7 Heck L.C., Cony F.G., Bianchi M.V., Driemeier D., Sonne L. \& Pavarini S.P. 2018. Pathological features of 78 metastatic or multicentric neoplasms involving the central nervous system in dogs. Pesquisa Veterinária Brasileira. 38(10): 1989-1998. 
8 Kim J.H., Kim W.J., Park J., Shin J.I. \& Yoon H.Y. 2015. Canine mammary anaplastic carcinoma with concurrent aorto-iliac thrombosis in a dog: a case report. Veterinarni Medicina. 60(7): 391-398.

9 Laforcade A.M., Freeman L.M., Shaw S.P., Brooks M.B., Rozanski E.A. \& Rush J.E. 2003. Hemostatic Changes in Dogs with Naturally Occurring Sepsis. Journal of Veterinary Internal Medicine. 17: 674-679.

10 Laforcade A. 2012. Disease associated to thrombosis. Topics in Companion Animal Medicine. 27: 59-64.

11 Laurenson M.P., Hopper K., Herrera M.A. \& Johnson E.G. 2010. Concurrent diseases and conditions in dogs with splenic vein thrombosis. Journal of Veterinary Internal Medicine. 24: 1298-1304.

12 Maali Y., Badiou C., Martins-Simões P., Hodille E., Bes M., Vandenesch F., Lina G., Diot A., Laurent F. \& Trouillet-Assant S. 2018. Understanding the Virulence of Staphylococcus pseudintermedius: A Major Role of PoreForming Toxins. Frontiers in Cellular and Infection Microbiology. 8: 221. 